\title{
Exponential sums with coefficients of certain Dirichlet series
}

\section{Stephan Baier}

Received: 1 September 2011 / Accepted: 13 December 2011 / Published online: 23 December 2011

C The Author(s) 2011. This article is published with open access at Springerlink.com

\begin{abstract}
Under the generalized Lindelöf Hypothesis in the $t$ - and $q$-aspects, we bound exponential sums with coefficients of Dirichlet series belonging to a certain class. We use these estimates to establish a conditional result on squares of Hecke eigenvalues at Piatetski-Shapiro primes.
\end{abstract}

Keywords Exponential sums · Dirichlet series · Hecke eigenvalues ·

Piatetski-Shapiro primes

Mathematics Subject Classification (2000) $\quad 11 \mathrm{~F} 11 \cdot 11 \mathrm{~F} 30 \cdot 11 \mathrm{~L} 07 \cdot 11 \mathrm{M} 99$

\section{General assumptions}

In this paper, we derive a conditional estimate for exponential sums of the form

$$
\sum_{n \sim N} a_{n} e(f(n))
$$

where $a_{n}$ is the $n$th coefficient of Dirichlet series $F(s)$ whose twists with Dirichlet characters satisfy the generalized Lindelöf Hypothesis in the $t$ - and $q$-aspects, and $f(x)$ is a function having certain properties. As an application, we consider squares of Hecke eigenvalues at Piatetski-Shapiro primes. In the following, we state the required conditions on $F(s)$ and $f(x)$.

Communicated by J. Schoißengeier.

S. Baier $(\bowtie)$

Mathematisches Institut, Universität Göttingen, Bunsenstr. 3-5, 37073 Göttingen, Germany

e-mail: sbaier@uni-math.gwdg.de 
Conditions on the $L$-function We assume that

$$
F(s)=\sum_{n=1}^{\infty} a_{n} n^{-s}
$$

is a Dirichlet series, absolutely convergent for $\Re s>1$, which satisfies the following conditions (a), (b) and (c).

(a) $F(s)$ lies in the extended Selberg class of Dirichlet series which don't necessarily possess a functional equation (for the definition of the Selberg class, see [6], Sect. 8.1, for example), i.e. $F(s)$ has the following properties.

(i) (Analyticity) There exists some $m \in \mathbb{N}$ such that $(s-1)^{m} F(s)$ extends to an entire function of finite order.

(ii) (Ramanujan conjecture) $a_{1}=1$ and $a_{n} \ll_{\varepsilon} n^{\varepsilon}$ for any $\varepsilon>0$.

(iii) (Euler product) For $\Re s>1$, the function $F(s)$ can be written as a product over primes in the form

$$
F(s)=\prod_{p} F_{p}(s),
$$

where $\log F_{p}(s)$ is a Dirichlet series of the form

$$
\log F_{p}(s)=\sum_{n=0}^{\infty} b_{p^{k}} p^{-k s}
$$

with complex coefficients $b_{p^{k}}$ satisfying

$$
b_{p^{k}}=O\left(p^{k \theta}\right)
$$

for some $\theta<1 / 2$.

(b) For any Dirichlet character $\chi$ define

$$
F(s, \chi):=\sum_{n=1}^{\infty} a_{n} \chi(n) n^{-s} \text { for } \Re s>1 .
$$

Then $(s-1)^{m} F(s, \chi)$ extends to an entire function again.

(c) The family of functions $F(s, \chi)$ satisfies the Lindelöf Hypothesis in the $t$ - and $q$-aspects, i.e.

$$
F\left(\frac{1}{2}+i t, \chi\right) \ll|t q|^{\varepsilon} \quad \text { for all }|t| \geqslant 1, \quad q \in \mathbb{N} \text { and characters } \chi \bmod q .
$$

Conditions on $f \quad$ We assume that $f:[1, \infty) \rightarrow \mathbb{R}^{+}$satisfies the following conditions (a)-(f).
(a) $f$ is three times continuously differentiable.
(b) $f$ is monotonically increasing.
(c) $f(x) \asymp f(2 x)$ for all $x \geqslant 1$.
(d) $f^{(k)}(x) \asymp f(x) / x^{k}$ for all $x \geqslant 1$ and $k=1,2,3$. 
(e) $f^{\prime}(x)+x f^{\prime \prime}(x) \asymp f(x) / x$ for all $x \geqslant 1$.

(f) $2 f^{\prime \prime}(x)+x f^{\prime \prime \prime}(x) \asymp f(x) / x^{2}$ for all $x \geqslant 1$.

A typical example of a function of this type is

$$
f(x)=A x^{\gamma},
$$

where $A>0$ and $\gamma \neq 0$ are constants. This function will come up in connection with our application to squares of Hecke eigenvalues at Piatetski-Shapiro primes (see Sect. 10).

We shall also use the usual convention that $\varepsilon$ is an arbitrarily small positive number and that the implied constants in all estimates involving $\varepsilon$ may depend on $\varepsilon$.

\section{Results}

Our first result is the following.

Theorem 1 Fix $\eta>0$. Suppose that $1 \leqslant N<N^{\prime} \leqslant 2 N$ and

$$
N^{1 / 2+\eta} \leqslant f(N) \leqslant N^{3 / 2-\eta} .
$$

Then, under the conditions in Sect. 1, we have

$$
\sum_{N<n \leqslant N^{\prime}} a_{n} e(f(n)) \ll_{f, \eta, \varepsilon} N^{19 / 22+\varepsilon} f(N)^{1 / 11} .
$$

We note that the above bounds are non-trivial if $\varepsilon<\eta / 11$ and $N$ is large enough. With applications in mind, we also prove the following modification of Theorem 1.

Theorem 2 Let $m \in \mathbb{N}$. Then, under the conditions in Theorem 1 and Sect. 1, we have

$$
\sum_{\substack{n \sim N \\(n, m)=1}} \mu^{2}(n) a_{n} e(f(n)) \ll_{f, \eta, \varepsilon} m^{\varepsilon} N^{19 / 22+\varepsilon} f(N)^{1 / 11} .
$$

Let now $G$ be a Hecke eigenform of weight $\kappa$ for the full modular group $\operatorname{SL}_{2}(\mathbb{Z})$. By $\lambda(n)$ we denote the normalized $n$th Fourier coefficient of $G$, i.e.

$$
G(z)=\sum_{n=1}^{\infty} \lambda(n) n^{(\kappa-1) / 2} e(n z) \text { for } \Im z>0, \text { and } \lambda(1)=1
$$

These Hecke eigenvalues satisfy the Ramanujan conjecture $\lambda(n) \ll_{\varepsilon} n^{\varepsilon}$ and the multiplicative property

$$
\lambda(m n)=\sum_{d \mid \operatorname{gcd}(m, n)} \mu(d) \lambda\left(\frac{m}{d}\right) \lambda\left(\frac{n}{d}\right) \quad \text { for all } m, n \in \mathbb{N},
$$


which follows from the well-known formula

$$
\lambda(m) \lambda(n)=\sum_{d \mid \operatorname{gcd}(m, n)} \lambda\left(\frac{m n}{d^{2}}\right)
$$

by Möbius inversion.

Let $L\left(\operatorname{Sym}^{2} G, s\right)$ be the symmetric square $L$-function for $G$, defined by

$$
L\left(\operatorname{Sym}^{2} G, s\right)=\zeta(2 s) \sum_{n=1}^{\infty} \lambda\left(n^{2}\right) n^{-s} \text { for } \Re s>1
$$

We note that by multiplying out the right-hand side, we get

$$
L\left(\operatorname{Sym}^{2} G, s\right)=\sum_{n=1}^{\infty} a_{n} n^{-s} \text { for } \Re s>1,
$$

where the coefficients of the Dirichlet series on the right-hand side satisfy $a_{n}=\lambda\left(n^{2}\right)$ for any squarefree $n$. Moreover, it is well-known that $L\left(\operatorname{Sym}^{2} G, s\right)$ lies in the Selberg class and hence satisfies condition a) in Sect. 1.

More generally, for any Dirichlet character $\chi$ let $L\left(\operatorname{Sym}^{2} G \otimes \chi, s\right)$ be the symmetric square $L$-function for $G$ twisted with $\chi$, defined by

$L\left(\operatorname{Sym}^{2} G \otimes \chi, s\right)=\sum_{n=1}^{\infty} \chi(n) a_{n} n^{-s}=L\left(2 s, \chi^{2}\right) \sum_{n=1}^{\infty} \chi(n) \lambda\left(n^{2}\right) n^{-s}$ for $\Re s>1$.

As a consequence of the work of Shimura [7], $L\left(\operatorname{Sym}^{2} G \otimes \chi, s\right)$ extends analytically to the whole complex plane and hence satisfies condition b) in Sect. 1. If $\chi$ is primitive, then $L\left(\operatorname{Sym}^{2} G \otimes \chi, s\right)$ even lies in the Selberg class.

The Lindelöf Hypothesis in the $t$ - and $q$-aspects for the family of $L$-functions $L\left(\operatorname{Sym}^{2} G \otimes \chi, s\right)$, with $G$ fixed, asserts that

$L\left(\operatorname{Sym}^{2} G \otimes \chi, \frac{1}{2}+i t\right) \ll(t q)^{\varepsilon}$ for all $|t| \geqslant 1, q \in \mathbb{N}$ and characters $\chi \bmod q$.

We note that it can be deduced from Theorem 1 in [3] that (2.4) holds if $L\left(\operatorname{Sym}^{2} G \otimes \chi, s\right)$ satisfies the Riemann Hypothesis for all primitive characters $\chi$.

In [1], we bounded the average of $\lambda(p)$ at Piatetski-Shapiro primes, i.e. primes of the form $p=\left[n^{c}\right]$ with $n \in \mathbb{N}$ and $c>1$ fixed. The $c$-range for which we obtained a non-trivial result was $1<c<8 / 7$. In this range, we proved that there exists a constant $C>0$ possibly depending on $G$ such that

$$
\sum_{\substack{n \leqslant N \\\left[n^{c}\right] \in \mathbb{P}}} \lambda\left(\left[n^{c}\right]\right) \ll_{G, c} N \exp (-C \sqrt{\log N}),
$$


where here as in the sequel, $\mathbb{P}$ is the set of primes. We posed the question if also an asymptotic estimate for the average of the squares of these Hecke eigenvalues at Piatetski-Shapiro can be established. Employing Theorem 2, we shall prove the following conditional result.

Theorem 3 Let $1<c<25 / 24$ be fixed and $\mathbb{P}$ be the set of primes. Assume that (2.4) holds. Then we have

$$
\sum_{\substack{n \leqslant N \\\left[n^{c}\right] \in \mathbb{P}}} \lambda\left(\left[n^{c}\right]\right)^{2} \sim \frac{N}{c \log N} \text { as } N \rightarrow \infty .
$$

According to [1], Theorem 3 and (2.5) imply the following result on the sign changes of $\lambda(p)$ at Piatetski-Shapiro primes $p$.

Theorem 4 Let $1<c<25 / 24$ be fixed and assume that (2.4) holds. Then $\lambda(p)$ changes sign infinitely often as $p$ runs through the primes of the form $p=\left[n^{c}\right]$ with $n \in \mathbb{N}$.

We point out that the full strength of the Lindelöf hypothesis is not required to obtain non-trivial bounds for the exponential sums in question. However, in this paper, we want to establish the strongest possible result that our method allows.

\section{Farey dissection}

Our goal is to establish a non-trivial bound for the exponential sum

$$
\sum_{n \sim N} a_{n} e(f(n))
$$

in Theorem 1. To this end, we shall split this exponential sum into short subsums using a Farey dissection of a certain interval. We note that the splitting of the summation interval in the present paper differs from that in [1]. It will become clear in the next section why it is advantageous to split the summation interval as described below.

For $x \geqslant 1$, let

$$
h(x):=f^{\prime}(x)+x f^{\prime \prime}(x) .
$$

By the condition (f) on $f$ in Sect. 1, we have

$$
h^{\prime}(x)=2 f^{\prime \prime}(x)+x f^{\prime \prime \prime}(x) \asymp \frac{f(x)}{x^{2}} .
$$

Since $f$ is assumed to take only positive values and $h^{\prime}$ is continuous, it follows that $h^{\prime}(x)$ doesn't change sign. Hence, $h(x)$ is monotonically increasing or decreasing. In 
the sequel, we assume without loss of generality that $h(x)$ is monotonically decreasing (in particular, if $f(x)$ is defined as in (10.7) in Sect. 10, then $h(x)$ will have this property). Let $Q$ be a real parameter with

$$
1 \leqslant Q \leqslant N
$$

to be chosen later.

Now we make a Farey dissection of level $Q$ of the interval $\left[h\left(N^{\prime}\right), h(N)\right.$ ) (for details on Farey intervals, see [1], for example). In this way, we write $\left[h\left(N^{\prime}\right), h(N)\right)$ as the disjoint union of intervals of the form

$$
\left[\frac{l}{q}-\frac{M_{1}}{q Q}, \frac{l}{q}+\frac{M_{2}}{q Q}\right) \cap\left[h\left(N^{\prime}\right), h(N)\right),
$$

where $M_{1}, M_{2} \asymp 1, q \leqslant Q$ and $(q, l)=1$. Projecting these intervals back into $\left(N, N^{\prime}\right]$ under the map $h^{-1}$, we get intervals of the form

$h^{-1}\left(\left[\frac{l}{q}-\frac{M_{1}}{q Q}, \frac{l}{q}+\frac{M_{2}}{q Q}\right) \cap\left[h\left(N^{\prime}\right), h(N)\right)\right)=\left(x_{0}-m_{1}, x_{0}+m_{2}\right] \subseteq\left(N, N^{\prime}\right]$

with

$$
x_{0}=h^{-1}\left(\frac{l}{q}\right)
$$

and

$$
m_{1}, m_{2} \ll \frac{1}{q Q} \cdot\left(h^{-1}\right)^{\prime}\left(\frac{l}{q}\right)=\frac{1}{q Q} \cdot \frac{1}{h^{\prime}\left(x_{0}\right)} \asymp \frac{N^{2}}{q Q f(N)},
$$

by (3.2) and the conditions b) and c) on $f$ in Sect. 1 .

In the following sections, we shall estimate the subsums

$$
\sum_{x_{0}-m_{1}<n \leqslant x_{0}+m_{2}} a_{n} e(f(n)) .
$$

\section{Approximation of $f$ and partial summation}

In $\left(x_{0}-m_{1}, x_{0}+m_{2}\right]$, we now approximate the function $f(x)$ by

$$
g(x)=h\left(x_{0}\right) x-x_{0}^{2} f^{\prime \prime}\left(x_{0}\right) \log x+C=\frac{l}{q} \cdot x-x_{0}^{2} f^{\prime \prime}\left(x_{0}\right) \log x+C,
$$

where

$$
C:=f\left(x_{0}\right)-h\left(x_{0}\right) x_{0}+x_{0}^{2} f^{\prime \prime}\left(x_{0}\right) \log x_{0} .
$$


Using the definition of $h(x)$ in (3.1), It follows that

$$
g\left(x_{0}\right)=f\left(x_{0}\right), \quad g^{\prime}\left(x_{0}\right)=f^{\prime}\left(x_{0}\right), \quad \text { and } \quad g^{\prime \prime}\left(x_{0}\right)=f^{\prime \prime}\left(x_{0}\right) .
$$

Hence, applying Taylor's theorem to approximate $(f-g)^{\prime}(x)$ near $x_{0}$, we have

$$
f^{\prime}(x)-g^{\prime}(x)=\frac{1}{2}\left(x-x_{0}\right)^{2}\left(f^{\prime \prime \prime}(c)-g^{\prime \prime \prime}(c)\right)
$$

for some $c \in\left[x_{0}-m_{1}, x_{0}+m_{2}\right]$ if $x \in\left(x_{0}-m_{1}, x_{0}+m_{2}\right]$. Now,

$$
f^{\prime \prime \prime}(c)-g^{\prime \prime \prime}(c)=f^{\prime \prime \prime}(c)-\frac{2 x_{0}^{2} f^{\prime \prime}\left(x_{0}\right)}{c^{3}} \ll \frac{f(N)}{N^{3}},
$$

by our conditions on $f$. Hence,

$$
f^{\prime}(x)-g^{\prime}(x) \ll \frac{N^{4}}{q^{2} Q^{2} f(N)^{2}} \cdot \frac{f(N)}{N^{3}} \ll \frac{N}{q^{2} Q^{2} f(N)} .
$$

Using partial summation, we deduce that

$$
\begin{aligned}
& \sum_{n \in\left(x_{0}-m_{1}, x_{0}+m_{2}\right]} a_{n} e(f(n)) \\
= & \sum_{n \in\left(x_{0}-m_{1}, x_{0}+m_{2}\right]} a_{n} e(g(n)) e(f(n)-g(n)) \\
= & e\left(f\left(x_{0}+m_{2}\right)-g\left(x_{0}+m_{2}\right)\right) \sum_{n \in\left(x_{0}-m_{1}, x_{0}+m_{2}\right]} a_{n} e(g(n)) \\
& -2 \pi i \int_{x_{0}-m_{1}}^{x_{0}+m_{2}}\left(\sum_{n \in\left(x_{0}-m_{1}, u\right]} a_{n} e(g(n))\right)\left(f^{\prime}(u)-g^{\prime}(u)\right) e(f(u)-g(u)) \mathrm{d} u \\
\ll & \left(1+\left(m_{1}+m_{2}\right) \cdot \frac{N}{q^{2} Q^{2} f(N)}\right) \cdot \max _{u \leqslant x_{0}+m_{2}}\left|\sum_{n \in\left(x_{0}-m_{1}, u\right]} a_{n} e(g(n))\right| \\
\ll & \left(1+\frac{N^{3}}{q^{3} Q^{3} f(N)^{2}}\right) \cdot \max _{u \leqslant x_{0}+m_{2}}\left|\sum_{n \in\left(x_{0}-m_{1}, u\right]} a_{n} e(g(n))\right|
\end{aligned}
$$

Thus we have replaced the function $f(x)$ by $g(x)$. The exponential sum with $g(n)$ in place of $f(n)$ can now be related to the functions $F(s, \chi)$. This will be done in the next sections.

In [1], we approximated the function $f(x)$ just by a linear function of the form

$$
g(x)=\frac{l}{q} \cdot x+C
$$


in an interval around the point $x_{0}=f^{\prime-1}(l / q)$. However, in this way one can just force the first derivative of $g(x)$ to agree with that of $f(x)$ at the point at $x=x_{0}$. The approximation of $f(x)$ by the function $g(x)$ defined in (4.1) allows to force the first and the second derivatives of $g(x)$ and $f(x)$ to agree at $x=x_{0}$. This reduces the error in the approximation substantially and is the key point of this paper.

\section{Rewriting $\sum_{n} a_{n} e(g(n))$ using multiplicative characters}

We have

$$
\sum_{x_{0}-m_{1}<n \leqslant u} a_{n} e(g(n))=e(C) \cdot \sum_{x_{0}-m_{1}<n \leqslant u} a_{n} e\left(n \cdot \frac{l}{q}\right) \cdot n^{-i T}
$$

with

$$
T:=2 \pi x_{0}^{2} f^{\prime \prime}\left(x_{0}\right)
$$

We break the sum over $n$ as follows.

$$
\begin{aligned}
\sum_{x_{0}-m_{1}<n \leqslant u} a_{n} e\left(n \cdot \frac{l}{q}\right) \cdot n^{-i T} & =\sum_{d \mid q} \sum_{\substack{x_{0}-m_{1}<n \leqslant u \\
(n, q)=d}} a_{n} e\left(n \cdot \frac{l}{q}\right) \cdot n^{-i T} \\
& =\sum_{d \mid q} d^{-i T} \sum_{\substack{\left(x_{0}-m_{1}\right) / d<n \leqslant u / d \\
(n, q / d)=1}} a_{d n} e\left(n \cdot \frac{l}{q / d}\right) \cdot n^{-i T} .
\end{aligned}
$$

Now we write the additive character in the last line using multiplicative characters in the form

$$
e\left(n \cdot \frac{l}{q / d}\right)=\frac{1}{\varphi(q / d)} \cdot \sum_{\chi \bmod q / d} \bar{\chi}(l) \tau(\bar{\chi}) \chi(n) .
$$

It follows that

$$
\begin{aligned}
& \sum_{x_{0}-m_{1} \leqslant n \leqslant u} a_{n} e\left(n \cdot \frac{l}{q}\right) \cdot n^{-i T} \\
= & \sum_{d \mid q} d^{-i T} \sum_{\chi \bmod q / d} \frac{1}{\varphi(q / d)} \cdot \bar{\chi}(l) \tau(\bar{\chi}) \sum_{\left(x_{0}-m_{1}\right) / d<n \leqslant u / d} a_{d n} \chi(n) n^{-i T} .
\end{aligned}
$$

\section{Reduction to $F(s, \chi)$}

Using Perron's formula (see [2], Lemma 1.4.2., for example) and the Ramanujan conjecture, $a_{n} \ll n^{\varepsilon}$, we have 


$$
\begin{aligned}
\sum_{\left(x_{0}-m_{1}\right) / d<n \leqslant u / d} a_{d n} \chi(n) n^{-i T}= & \int_{c-i T_{0}}^{c+i T_{0}}\left(\sum_{n=1}^{\infty} a_{d n} \chi(n) n^{-s-i T}\right) \\
& \times\left(\left(\frac{u}{d}\right)^{s}-\left(\frac{x_{0}-m_{1}}{d}\right)^{s}\right) \frac{\mathrm{d} s}{s} \\
& +O\left(\frac{N^{1+\varepsilon}}{d T_{0}}+N^{\varepsilon}\right)
\end{aligned}
$$

for $c=1+1 / \log N$ and $T_{0} \geqslant 1$, where we recall that

$$
N \leqslant x_{0}-m_{1}<u \leqslant N^{\prime} \leqslant 2 N .
$$

Next, we relate the Dirichlet series in the integrand to $F(s, \chi)$.

Since $F(s)$ has an Euler product, the coefficients $a_{n}$ of $F(s)$ are multiplicative in $n$. Hence, for $\Re s>1$, we have

$$
\sum_{n=1}^{\infty} a_{d n} \chi(n) n^{-s}=\left(\sum_{\substack{n=1 \\ s(n) \mid d}}^{\infty} a_{d n} \chi(n) n^{-s}\right) \cdot\left(\sum_{\substack{n=1 \\(n, d)=1}}^{\infty} a_{n} \chi(n) n^{-s}\right)
$$

where $s(n)$ is the largest squarefree number dividing $n$, and we may write

$$
\sum_{\substack{n=1 \\ s(n) \mid d}}^{\infty} a_{d n} \chi(n) n^{-s}=\prod_{p \mid d} \sum_{k=0}^{\infty} a_{p^{\alpha(p)+k}} \chi^{k}(p) p^{-k s},
$$

where

$$
d=\prod_{p \mid d} p^{\alpha(p)}
$$

is the prime number factorization of $d$. Further,

$$
\sum_{\substack{n=1 \\(n, d)=1}}^{\infty} a_{n} \chi(n) n^{-s}=F(s, \chi) \prod_{p \mid d}\left(\sum_{k=0}^{\infty} a_{p^{k}} \chi^{k}(p) p^{-k s}\right)^{-1}
$$

So altogether,

$$
\sum_{n=1}^{\infty} a_{d n} \chi(n) n^{-s}=G_{d}(s, \chi) F(s, \chi)
$$


with

$$
G_{d}(s, \chi)=\prod_{p \mid d} \frac{\sum_{k=0}^{\infty} a_{p^{\alpha(p)+k}} \chi^{k}(p) p^{-k s}}{\sum_{k=0}^{\infty} a_{p^{k}} \chi^{k}(p) p^{-k s}}
$$

Hence, the integral on the right-hand side of (6.1) takes the form

$$
\begin{aligned}
& \int_{c-i T_{0}}^{c+i T_{0}}\left(\sum_{n=1}^{\infty} a_{d n} \chi(n) n^{-s-i T}\right)\left(\left(\frac{u}{d}\right)^{s}-\left(\frac{x_{0}-m_{1}}{d}\right)^{s}\right) \frac{\mathrm{d} s}{s} \\
& =\int_{c-i T_{0}}^{c+i T_{0}} G_{d}(s+i T, \chi) F(s+i T, \chi)\left(\left(\frac{u}{d}\right)^{s}-\left(\frac{x_{0}-m_{1}}{d}\right)^{s}\right) \frac{\mathrm{d} s}{s} .
\end{aligned}
$$

\section{Estimation of the integral}

We shall need a bound for $G_{d}(s, \chi)$ if $\Re s \geqslant 1 / 2$, which we establish in the following. By the Ramanujan conjecture, $a_{n} \ll n^{\varepsilon}$, we have

$$
\sum_{k=0}^{\infty} a_{p^{\alpha(p)+k}} \chi^{k}(p) p^{-k s} \ll p^{\alpha(p) \varepsilon} \quad \text { uniformly for } \Re s \geqslant \frac{1}{2} .
$$

Let $t_{0} \in \mathbb{R}$ such that

$$
p^{-i t_{0}}=\chi(p)
$$

Then

$$
\sum_{k=0}^{\infty} a_{p^{k}} \chi^{k}(p) p^{-k s}=\sum_{k=0}^{\infty} a_{p^{k}} p^{-k\left(s+i t_{0}\right)},
$$

and by axiom (iii) (Euler product) for $F(s)$, we therefore have

$$
\log \sum_{k=0}^{\infty} a_{p^{k}} \chi^{k}(p) p^{-k s}=\log F_{p}\left(s+i t_{0}\right)=\sum_{k=0}^{\infty} b_{p^{k}} p^{-k\left(s+i t_{0}\right)}=\sum_{k=0}^{\infty} b_{p^{k}} \chi^{k}(p) p^{-k s}
$$

where $b_{p^{k}}(k=0,1, \ldots)$ are suitable coefficients satisfying

$$
b_{p^{k}} \ll p^{k \theta}
$$


for some $\theta<1 / 2$. It follows that

$$
\left(\sum_{k=0}^{\infty} a_{p^{k}} \chi^{k}(p) p^{-k s}\right)^{-1}=O(1) \text { uniformly for } \Re s \geqslant 1 / 2
$$

From the above estimates, we deduce that

$$
G_{d}(s, \chi) \ll d^{\varepsilon} \quad \text { uniformly for } \Re s \geqslant 1 / 2 \text {. }
$$

Furthermore, using conditions (b) and (c) on $F(s, \chi)$ in Sect. 1 together with the Phragmen-Lindelöf principle, we have

$$
F(\sigma+i t, \chi) \ll(t q)^{\varepsilon} \quad \text { uniformly for } \sigma \geqslant \frac{1}{2} \text { and }|\sigma+i t-1| \geqslant \frac{1}{4} .
$$

Thus,

$$
G_{d}(\sigma+i t, \chi) F(\sigma+i t, \chi) \ll(d t q)^{\varepsilon} \quad \text { uniformly for } \sigma \geqslant \frac{1}{2} \text { and }|\sigma+i t-1| \geqslant \frac{1}{4} \text {. }
$$

Now we bound the integral on the right-hand side of (6.3), where we suppose that

$$
T_{0} \leqslant \frac{T}{2}
$$

Using Cauchy's integral theorem, we then have

$$
\begin{aligned}
& \int_{c-i T_{0}}^{c+i T_{0}} G_{d}(s+i T, \chi) F(s+i T, \chi)\left(\left(\frac{u}{d}\right)^{s}-\left(\frac{x_{0}-m_{1}}{d}\right)^{s}\right) \frac{\mathrm{d} s}{s} \\
& =\left(\int_{c-i T_{0}}^{1 / 2-i T_{0}}+\int_{1 / 2-i T_{0}}^{1 / 2+i T_{0}}+\int_{1 / 2+i T_{0}}^{c+i T_{0}}\right) G_{d}(s+i T, \chi) F(s+i T, \chi) \\
& \quad \times\left(\left(\frac{u}{d}\right)^{s}-\left(\frac{x_{0}-m_{1}}{d}\right)^{s}\right) \frac{\mathrm{d} s}{s} .
\end{aligned}
$$

From (6.2), (7.2), (7.3), (7.4) and $d \mid q$, we deduce that

$$
\begin{aligned}
& \int_{c-i T_{0}}^{c+i T_{0}} G_{d}(s+i T, \chi) F(s+i T, \chi)\left(\left(\frac{u}{d}\right)^{s}-\left(\frac{x_{0}-m_{1}}{d}\right)^{s}\right) \frac{\mathrm{d} s}{s} \\
& \ll(T q)^{\varepsilon} \cdot\left(\left(\frac{N}{d}\right)^{1 / 2}+\frac{N}{d T_{0}}\right)
\end{aligned}
$$

if $T \geqslant 1 / 2$. 


\section{Proof of Theorem 1}

Now we choose

$$
T_{0}:=\left(\frac{N}{d}\right)^{1 / 2}
$$

We note that

$$
T \asymp f(N)
$$

by (5.2), $x_{0} \asymp N$ and our conditions of $f$. Hence, by (2.1), the condition (7.3) is satisfied if $N$ is large enough. From (2.1), (6.1), (6.3), (7.5), (8.1), (8.2) and $d \leqslant N$ (by $d \mid q, q \leqslant Q$ and (3.3)), we deduce that

$$
\sum_{\left(x_{0}-m_{1}\right) / d<n \leqslant u / d} a_{d n} \chi(n) n^{-i T} \ll(q N)^{\varepsilon} \cdot\left(\frac{N}{d}\right)^{1 / 2} .
$$

Plugging this into (5.3), and using $\varphi(q / d) \gg q^{1-\varepsilon} / d$ and $|\tau(\bar{\chi})| \leqslant \sqrt{q / d}$, we obtain

$$
\left|\sum_{x_{0}-m_{1}<n \leqslant u} a_{n} e\left(n \cdot \frac{l}{q}\right) n^{-i T}\right| \ll(q N)^{1 / 2+\varepsilon}
$$

This together with (4.2) and (5.1) yields

$$
\sum_{n \in\left(x_{0}-m_{1}, x_{0}+m_{2}\right]} a_{n} e(f(n)) \ll\left(1+\frac{N^{3}}{q^{3} Q^{3} f(N)^{2}}\right)(q N)^{1 / 2+\varepsilon} .
$$

In Sect. 3, we have divided the interval $\left[h\left(N^{\prime}\right), h(N)\right)$ into Farey intervals around fractions $l / q$ with

$$
1 \leqslant q \leqslant Q, \quad l \asymp q \cdot h(N) \asymp q \cdot \frac{f(N)}{N} \quad \text { and } \quad(q, l)=1 .
$$

Hence, summing the contributions of the short sums in (8.4) over all relevant $q$ and $l$, we get

$$
\begin{aligned}
\sum_{n \sim N} a_{n} e(f(n)) & \ll \sum_{q \leqslant Q} \sum_{l \asymp q f(N) / N}\left(1+\frac{N^{3}}{q^{3} Q^{3} f(N)^{2}}\right)(q N)^{1 / 2+\varepsilon} \\
& \ll\left(\frac{Q^{5 / 2} f(N)}{N^{1 / 2}}+\frac{N^{5 / 2}}{Q^{3} f(N)}\right)(Q N)^{\varepsilon} .
\end{aligned}
$$


Now we choose

$$
Q:=\left(\frac{N^{5 / 2}}{f(N)} \cdot \frac{N^{1 / 2}}{f(N)}\right)^{2 / 11}=\frac{N^{6 / 11}}{f(N)^{4 / 11}}
$$

Thus we get

$$
\sum_{n \sim N} a_{n} e(f(n)) \ll N^{19 / 22+\varepsilon} f(N)^{1 / 11}
$$

which completes the proof.

\section{Proof of Theorem 2}

Theorem 2 can be proved along similar lines as Theorem 1. The arguments in Sects. 3, 4 and 5 carry over completely. We are then led to the sum

$$
\sum_{\substack{\left(x_{0}-m_{1}\right) / d<n \leqslant t / d \\(n, m)=1}} \mu^{2}(d n) a_{d n} \chi(n) n^{-i T}
$$

in place of the sum

$$
\sum_{\left(x_{0}-m_{1}\right) / d<n \leqslant t / d} a_{d n} \chi(n) n^{-i T}
$$

considered in Sects. 6,7 and 8. We now use the fact that $a_{n}$ is multiplicative in $n$ to rewrite the sum in question in the form

$\sum_{\substack{\left(x_{0}-m_{1}\right) / d<n \leqslant t / d \\(n, m)=1}} \mu^{2}(d n) a_{d n} \chi(n) n^{-i T}=\mu^{2}(d) a_{d} \sum_{\left(x_{0}-m_{1}\right) / d<n \leqslant t / d} \mu^{2}(n) a_{n} \chi_{1}(n) n^{-i T}$,

where $\chi_{1}(n)=\chi(n) \chi_{0}(n), \chi_{0}(n)$ being the principal character modulo $d m$. Similarly as in Sect. 6, we relate the sum over $n$ on the right-hand side to the corresponding Dirichlet series, which we write in the form

$$
\sum_{n=1}^{\infty} \mu^{2}(n) a_{n} \chi_{1}(n) n^{-s}=\prod_{p}\left(1+a_{p} \chi_{1}(p) p^{-s}\right)=H\left(s, \chi_{1}\right) F\left(s, \chi_{1}\right)
$$

where

$$
H\left(s, \chi_{1}\right)=\prod_{p} \frac{1+a_{p} \chi_{1}(p) p^{-s}}{\sum_{k=0}^{\infty} a_{p^{k}} \chi_{1}^{k}(p) p^{-k s}}=\prod_{p}\left(1-\frac{\sum_{k=2}^{\infty} a_{p^{k}} \chi_{1}^{k}(p) p^{-k s}}{\sum_{k=0}^{\infty} a_{p^{k}} \chi_{1}^{k}(p) p^{-k s}}\right) .
$$


By $a_{n} \ll n^{\varepsilon}$ and (7.1), the product on the right-hand side converges absolutely and uniformly in every compact subset $S$ of the half plane $\Re s>1 / 2$. Hence, the function $H\left(s, \chi_{1}\right)$ is entire there. Moreover, $\left|H\left(s, \chi_{1}\right)\right|$ is bounded by a constant $C(\varepsilon)$ if $\Re s \geqslant 1 / 2+\varepsilon$. The rest of the proof follows the arguments in the proof of Theorem 1 , where the function $G_{d}(s, \chi)$ is replaced by $H\left(s, \chi_{1}\right)$, and in the application of Cauchy's integral theorem, the line of integration is shifted to $\Re s=1 / 2+\varepsilon$ instead of $\Re s=1 / 2$.

\section{Proof of Theorem 3}

The general procedure of the proof will be similar as in [1], where we bounded the sum

$$
\sum_{\substack{n \leqslant N \\\left[n^{c}\right] \in \mathbb{P}}} \lambda\left(\left[n^{c}\right]\right)
$$

Therefore, we will be very brief in general and go into details only in the parts where the proof of Theorem 3 deviates substantially from that of Theorem 1 in [1]. First, we use the well-known relation

$$
\lambda(p)^{2}=1+\lambda\left(p^{2}\right)
$$

Hence, we have

$$
\sum_{\substack{n \leqslant N \\\left[n^{c}\right] \in \mathbb{P}}} \lambda\left(\left[n^{c}\right]\right)^{2}=\sum_{\substack{n \leqslant N \\\left[n^{c}\right] \in \mathbb{P}}} 1+\sum_{\substack{n \leqslant N \\\left[n^{c}\right] \in \mathbb{P}}} \lambda\left(\left[n^{c}\right]^{2}\right) .
$$

The ordinary Piatetski-Shapiro prime number theorem (see [1], for example) tells us that

$$
\sum_{\substack{n \leqslant N \\\left[n^{c}\right] \in \mathbb{P}}} 1 \sim \frac{N}{c \log N} \text { as } N \rightarrow \infty
$$

for every fixed $c$ in the range in Theorem 3. It remains to estimate the second sum on the right-hand side of (10.1). We write this sum in the form

$$
\sum_{\substack{n \leqslant N \\\left[n^{c}\right] \in \mathbb{P}}} \lambda\left(\left[n^{c}\right]^{2}\right)=\sum_{\substack{n \leqslant N \\\left[n^{c}\right] \in \mathbb{P}}} b_{\left[n^{c}\right]}
$$

where

$$
b_{n}:=\mu^{2}(n) \lambda\left(n^{2}\right)=\mu^{2}(n) a_{n},
$$

with $a_{n}$ as in (2.3). 
Clearly, it now suffices to bound the sum

$$
\sum_{n \leqslant N} b_{\left[n^{c}\right]} \Lambda\left(\left[n^{c}\right]\right)
$$

where $\Lambda(n)$ is the von Mangoldt function. Similarly as in [1], we pull out a main term which we estimate using an analogue of the prime number theorem for $\lambda\left(p^{2}\right)$. Then we reduce the error term to exponential sums as in [1] and treat the von Mangoldt function appearing in them using a Vaughan-type identity due to Heath-Brown (Lemma 4 in [1]). This leads to type I and type II sums. In [1], we then used the decomposition in (2.2) to separate the summation variables $m$ and $n$ in the said type I and type II sums. The decomposition of $b_{m n}$ needed here is simpler since we have

$$
b_{m n}= \begin{cases}0 & \text { if }(m, n)>1 \\ b_{m} b_{n} & \text { if }(m, n)=1\end{cases}
$$

due to the appearance of the Möbius function in the definition of $b_{n}$. Now the type I and type II sums take the form

$$
K=\sum_{h \sim H} \sum_{\substack{m \sim X \\(m, n)=1}} \sum_{n \sim Y} C_{h} A_{m} b_{n} e\left(h(m n)^{\gamma}\right)
$$

and

$$
L=\sum_{h \sim H} \sum_{\substack{m \sim X \\(m, n)=1}} \sum_{\substack{n \sim Y \\(m)}} C_{h} A_{m} B_{n} e\left(h(m n)^{\gamma}\right),
$$

where

$$
\gamma=\frac{1}{c}, \quad 1 \leqslant H \leqslant N^{1-\gamma+\eta} \text { and } X Y=N
$$

Here $C_{h}, A_{m}$ and $B_{n}$ are general coefficients of size $\ll N^{\varepsilon}$, and $b_{n}$ is defined as in (10.2).

We remove the coprimality condition $(m, n)=1$ in $K$ and $L$ using Möbius inversion, getting

$$
K=\sum_{d} \mu(d) K_{d} \quad \text { and } \quad L=\sum_{d} \mu(d) L_{d}
$$

with

$$
K_{d}=\sum_{h \sim H} \sum_{m \sim X / d} \sum_{n \sim Y / d} C_{h} A_{d m} b_{d n} e\left(h\left(d^{2} m n\right)^{\gamma}\right) .
$$


and

$$
L_{d}=\sum_{h \sim H} \sum_{m \sim X / d} \sum_{n \sim Y / d} C_{h} A_{d m} B_{d n} e\left(h\left(d^{2} m n\right)^{\gamma}\right) .
$$

Using (10.5) and Lemmas 15, 16 and 18 in [1], we deduce that

$$
L \ll N^{1-\eta} \text { if } N^{1-\gamma+100 \eta} \leqslant Y \leqslant N^{5 \gamma-4-100 \eta}
$$

and

$$
K \ll N^{1-\eta} \text { if } N^{3-3 \gamma+100 \eta} \leqslant Y \leqslant N^{\gamma-100 \eta},
$$

for some small $\eta>0$, provided that $\gamma>7 / 8$.

We note that if $0<\gamma<1$, then the function

$$
f(x)=h m^{\gamma} x^{\gamma}
$$

satisfies the conditions on $f$ in Sect. 1, and if $\gamma>1 / 2, h \sim H, m \sim X, Y \geqslant$ $N^{2 / 3+100 \eta}, \eta$ is sufficiently small and (10.4) is satisfied, then condition (2.1), with $N$ replaced by $Y$, in Theorems 1 and 2 holds, i.e.

$$
Y^{1 / 2+\eta} \leqslant f(Y) \leqslant Y^{3 / 2-\eta} .
$$

Now applying Theorem 2 with $f(n)$ defined as above and $a_{n}$ defined as in (2.3) to the inner sum over $n$ on the right-hand side of (10.3), we get

$$
\sum_{\substack{n \sim Y \\(m, n)=1}} b_{n} e\left(h(m n)^{\gamma}\right)=\sum_{\substack{n \sim Y \\(m, n)=1}} \mu^{2}(n) a_{n} e\left(h(m n)^{\gamma}\right) \ll H^{1 / 11} X^{\gamma / 11} Y^{19 / 22+\gamma / 11+\varepsilon}
$$

and hence

$$
K \ll H^{12 / 11} X^{1+\gamma / 11} Y^{19 / 22+\gamma / 11+\varepsilon},
$$

provided that $1 / 2<\gamma<1$ and $Y \geqslant N^{2 / 3+100 \eta}$. By (10.4), it follows that

$$
K \ll N^{1-\eta} \text { if } Y \geqslant N^{8-22 \gamma / 3+100 \eta},
$$

provided that $1 / 2<\gamma<1$. The $Y$-ranges in (10.6) and (10.8) overlap if $\gamma>24 / 25$ and $\eta$ is small enough. Hence, we have

$$
K \ll N^{1-\eta} \text { if } Y \geqslant N^{3-3 \gamma+100 \eta}
$$

provided that $24 / 25<\gamma<1$ and $\eta$ is sufficiently small. The rest of the proof is similar as in Sect. 12 in [1]. We note that the range $1<c<25 / 24$ in Theorem 3 comes from the above condition $24 / 25<\gamma<1$. 
Acknowledgments This work was supported by an ERC grant 258713.

Open Access This article is distributed under the terms of the Creative Commons Attribution Noncommercial License which permits any noncommercial use, distribution, and reproduction in any medium, provided the original author(s) and source are credited.

\section{References}

1. Baier, S., Zhao, L.: On Hecke eigenvalues at Piatetski-Shapiro primes. J. Lond. Math. Soc. (2) 81(1), 175-201 (2010)

2. Brüdern, J.: Einführung in die analytische Zahlentheorie. Springer, Berlin (1991)

3. Conrey, J.B., Ghosh, A.: Remarks on the generalized Lindelöf hypothesis. Funct. Approx. Comment. Math. 36, 71-78 (2006)

4. Deligne, P.: La conjecture de Weil I. Inst. Hautes Etudes Sci. Publ. Math. 43, 273-307 (1974)

5. Deligne, P.: La conjecture de Weil II. Inst. Hautes Etudes Sci. Publ. Math. 52, 137-252 (1980)

6. Goldfeld, D.: Automorphic forms and $L$-functions for the group $\operatorname{GL}(n, \mathbf{R})$. In: Cambridge Studies in Advanced Mathematics, vol. 99. Cambridge University Press, Cambridge (2006)

7. Shimura, G.: On the holomorphy of a certain Dirichlet series. Proc. Lond. Math. Soc. 31, 79-98 (1975) 